\title{
NUMERICAL MODELING OF COUPLED PARTIAL DIFFERENTIAL EQUATIONS USING RESIDUAL ERROR FUNCTIONS
}

\author{
Iryna Komashynska \\ Department of Mathematics \\ School of Science \\ The University of Jordan \\ Amman, 11942, JORDAN
}

\begin{abstract}
In this paper, the residual power series method is developed to solve a class of coupled partial differential equations. This approach improves solutions by reducing the residual error functions to create a rapidly convergent series. The description of the proposed method is presented to approximate the solution by highlighting all the steps necessary to implement the algorithm. Meanwhile, the scheme is tested on several cases of examples arising in the field of finance. Numerical results obtained justify that the proposed method is effective, accurate and simple in application.
\end{abstract}

AMS Subject Classification: 35A25, 35C10, 65D99

Key Words: coupled partial differential equations; residual power series method; approximate solution; Taylor expansion

\section{Introduction}

Mathematical models that use partial differential equations (PDEs) as the basis have become an integral part of research in most branches of science and engineering, and have recently also been expanded in economics and finance. PDE-based approaches are currently fairly standard as a pricing model in finance. In most cases, a model is described by coupled PDEs.

Received: April 11, 2020

(C) 2020 Academic Publications 
In this paper we concentrate on coupled PDEs, which arise in the pricing theory. In the last 50 years, various models were proposed for price options. One of the most significant model in option pricing is the Black-Scholes model [6], which involves a partial differential equation of parabolic type, so-called the Black-Scholes equation. The equation estimates the price of the option over time. However, this classical model do not reflect the true behavior of stock movements in the real world. A new regime-switching model was introduced,(see, for example [7]). This is due to the market may switch from time to time among a finite number of states "regimes". In practice, the changing between regimes is caused by different reasons, including shifts in economic policies, technological shifts, and so on. Obviously, the regime-switching model can better fit a market dynamics. This leads to incorporating of regime coupling terms in the system of PDEs describing the model.

We consider the American option pricing problem under regime switching. For a detailed description of the model, we refer to $[8,15]$. We just notice that the equations used in this model have the following form:

$$
\frac{\partial V_{i}}{\partial \tau}+\frac{1}{2} \sigma_{i}^{2} S^{2} \frac{\partial^{2} V_{i}}{\partial S^{2}}+r_{i} S \frac{\partial V_{i}}{\partial S}-r_{i} V_{i}+\sum_{j \neq i} q_{i j}\left(V_{j}-V_{i}\right)=0
$$

where $S$ is the asset price, $\tau$ is the time, $V(S, \tau)$ is the value of an American put option price in each regime $i, i=1,2, \cdots, n$, with striking price $K$. Here, let $\sigma_{i}, r_{i}$ and $q_{i j}$ be real constants in each regime.

Since there is no exact solution, various numerical and approximation methods have been employed including Fast Fourier Transform method [19, 24], finite difference method [10], method of lines [8, 14], penalty method [11], etc. In this work, we concentrate on coupled PDEs that arise in financial models using the residual power series method.

The residual power series method (RPSM) is based on the Taylor series expansion and the concept of a residual error function. It is efficient and convenient to use since it does not require discretization or linearization. The RPSM was first developed for solving first-order fuzzy differential equations. Later, it has been successfully applied to find numerical solutions for other equations, including ordinary and partial differential equations, nonlinear systems of singular initial value problems, pantograph delay differential equation, fractional differential equations, fuzzy fractional differential models $[1,2,3,4,5,12,13$, $16,17,18,20,21,22]$.

The rest of paper is organized as follows. Section 2 describes the basic concepts of the residual power series method. Application of this method for solving coupled system of PDEs is presented in Section 3.Numerical examples 
and analysis of the results are discussed in Section 4. Finally, conclusions are given in Section 5 .

\section{Fitted residual power series method}

In this section, we give a brief description of RPSM and extend of this technique for treating the system of coupled partial differential equations.

Consider the generalized system of coupled PDEs of the form:

$$
\frac{\partial V_{i}}{\partial t}+L V_{i}+F_{i}\left(V_{1}, V_{2}, \cdots, V_{n}\right)=0
$$

subject to the initial conditions

$$
V_{i}\left(x, t_{0}\right)=V_{i}^{0}(x), \quad i=1,2, \cdots, n,
$$

where $V_{i}(x, t), i=1,2, \cdots, n$, are unknown analytic functions on the given domain $D \subset \mathbb{R}^{2},(x, t) \in D . F_{i}$ are assumed to be analytic functions on $D$ and they denote a coupling term.

Let $L:=\frac{1}{2} \sigma_{i}^{2} x^{2} \frac{\partial^{2}}{\partial x^{2}}+r_{1} x \frac{\partial}{\partial x}-r_{1}$ be a partial differential operator.

According to RPSM [16-18], we assume that the solution of equations (2) and (3) has the following form of the power series:

$$
V_{i}(x, t)=\sum_{k=0}^{\infty} a_{i, k}(x)\left(t-t_{0}\right)^{k}, \quad i=1,2, \cdots, n .
$$

The solution can be approximated by the $m^{\text {th }}$-truncated series.

$$
V_{i}^{m}(x, t)=\sum_{k=0}^{m} a_{i, k}(x)\left(t-t_{0}\right)^{k}, \quad i=1,2, \cdots, n .
$$

By choosing the initial conditions (3), the initial guess of the approximation of system (2) and (3) can be given as

$$
a_{i, 0}(x)=V_{i}^{0}(x)
$$

Define the $m^{\text {th }}$ residual function as follows

$$
\operatorname{Res}_{i}^{m}(x, t)=\frac{\partial V_{i}^{m}}{\partial t}+L V_{i}^{m}+F_{i}\left(V_{1}^{m}, V_{2}^{m}, \cdots, V_{n}^{m}\right),
$$


and the residual function as

$$
\operatorname{Res}_{i}(x, t)=\lim _{m \rightarrow \infty} \operatorname{Res}_{i}^{m}(x, t) .
$$

Obviously, the residual function is infinite many times differentiable at $t=$ $t_{0}$ and $\operatorname{Res}_{i}(x, t)$ vanishes as $m$ approaches the infinity. In addition, we have

$$
\frac{\partial^{m-1}}{\partial t^{m-1}} \operatorname{Res}_{i}\left(x, t_{0}\right)=\frac{\partial^{m-1}}{\partial t^{m-1}} \operatorname{Res}_{i}^{m}\left(x, t_{0}\right)=0 \quad i=1,2, \cdots, n .
$$

Therefore, the value of coefficients $a_{i, k}(x), i=1,2, \cdots, n$, can be determined consecutively by solving the relation (3) with respect to $a_{i, k}(x), i=$ $1,2, \cdots, n$ at $t=t_{0}$. Furthermore, higher accuracy can be achieved by calculating more terms of the solution.

The next theorem, as a matter of fact, shows convergence of the RPSM to understand the behavior of the solution.

Theorem 2.1. Assume that $V_{i}(x, t), i=1,2, \cdots, n$, are the exact solutions for the system (2) and (3). Then, the approximate solutions obtained by $R P S M$ are in fact the Taylor expansion of $V_{i}(x, t)$ about $t=t_{0}$.

Proof. Suppose that the approximate solutions have the form

$$
\tilde{V}_{i}(x, t)=a_{i, 0}(x)+a_{i, 1}(x)\left(t-t_{0}\right)+a_{i, 2}(x)\left(t-t_{0}\right)^{2}+\cdots
$$

where $i=1,2, \cdots, n$.

Thus, it suffices to show that $a_{i, m}(x)=\frac{1}{m !} \frac{\partial^{m} V_{i}\left(x, t_{0}\right)}{\partial t^{m}}$, for each $m=0,1,2, \cdots, n$, where $V_{i}(x, t)$ are the exact solutions for (2) and (3).

For $m=0$, it follows from the initial conditions that

$$
a_{i, 0}(x)=V\left(x, t_{0}\right)=V_{i}^{0}(x), \quad i=1, \cdots, n .
$$

So,

$$
\tilde{V}_{i}(x, t)=V_{i}^{0}(x)+a_{i, 1}(x)\left(t-t_{0}\right)+\cdots
$$

To find $a_{i, 1}(x)(m=1)$, we substitute $t=t_{0}$ into (2) to obtain

$$
\frac{\partial V_{i}\left(x, t_{0}\right)}{\partial t}+\left[L V_{i}+F_{i}\right]\left(x, t_{0}\right)=0 .
$$

On the other hand, we substitute (12) into (2) and set $t=t_{0}$ as follows

$$
a_{i, 1}(x)+\left[L V_{i}+F_{i}\right]\left(x, t_{0}\right)=0 .
$$


Hence, by comparison, it follows that

$$
a_{i, 1}(x)=\frac{\partial V_{i}\left(x, t_{0}\right)}{\partial t}, \quad i=1,2, \cdots, n .
$$

Consequently, we have

$$
\tilde{V}_{i}=V_{i}^{0}(x)+\frac{\partial V_{i}\left(x, t_{0}\right)}{\partial t}\left(t-t_{0}\right)+a_{i, 2}(x)\left(t-t_{0}\right)^{2}+\cdots
$$

For $m=2$, differentiate both sides of Equation (2) with respect to $t$ to obtain that

$$
\frac{\partial^{2} V_{i}(x, t)}{\partial t^{2}}+\frac{\partial}{\partial t}\left[L V_{i}+F_{i}\right](x, t)=0, \quad i=1,2, \cdots, n .
$$

If $t=t_{0}$, we have

$$
\frac{\partial^{2} V_{i}\left(x, t_{0}\right)}{\partial t^{2}}+\frac{\partial}{\partial t}\left[L V_{i}+F_{i}\right]\left(x, t_{0}\right)=0, \quad 1=1,2, \cdots, n .
$$

Next, we substitute (16) into (17) for $t=t_{0}$, we obtain

$$
2 a_{i, 2}(x)+\frac{\partial}{\partial t}\left[L V_{i}+F_{i}\right]\left(x, t_{0}\right)=0, \quad 1=1,2, \cdots, n .
$$

Again, by comparison (18) and (19), we conclude that

$$
a_{i, 2}(x)=\frac{1}{2} \frac{\partial^{2} V_{i}\left(x, t_{0}\right)}{\partial t^{2}} .
$$

If we continue these calculations for $m=3,4, \cdots$, it can easily show that

$$
a_{i, m}(x)=\frac{1}{m !} \frac{\partial^{m} V_{i}\left(x, t_{0}\right)}{\partial t^{m}}, \quad i=1,2, \cdots, n .
$$

This completes the proof.

\section{Applications}

In this section, we present application of the proposed scheme -RPSM- for solving the system of coupled PDEs of the form

$$
\frac{\partial V_{1}}{\partial t}-\frac{1}{2} \sigma_{1}^{2} x^{2} \frac{\partial^{2} V_{1}}{\partial x^{2}}-r_{1} x \frac{\partial V_{1}}{\partial x}+r_{1} V_{1}+q_{1}\left(V_{1}-V_{2}\right)=0,
$$




$$
\frac{\partial V_{2}}{\partial t}-\frac{1}{2} \sigma_{2}^{2} x^{2} \frac{\partial^{2} V_{2}}{\partial x^{2}}-r_{2} x \frac{\partial V_{2}}{\partial x}+r_{2} V_{2}+q_{2}\left(V_{2}-V_{1}\right)=0,
$$

subject to the initial conditions

$$
V_{i}(x, 0)=f_{0}(x), \quad i=1,2 .
$$

This problem is a special case of the American option pricing problem under regime switching (1). We assume that, there are only two regimes $(i=1,2)$ and introduce a time-reverse transformation $t=T-\tau$. Also, for convenience, we rename the variable $S$ to $x$. In general, the American put option price $V_{i}(x, t)$ in each regime $i$ satisfies the free-boundary value problem. For computational purposes, Equation (22) is posed on the localized domain $D=\left[0, S_{\max }\right] \times[0, T]$, where $S_{\max }$ denotes a sufficiently large number to ensure the accuracy of the solution. The maturity $T$ and the strike $K$ are real constants.

The $m^{\text {th }}$-truncated series solutions have the following form:

$$
\begin{aligned}
& V_{1}^{m}(x, t)=\sum_{k=0}^{m} a_{k}(x) t^{k}, \\
& V_{2}^{m}(x, t)=\sum_{k=0}^{m} b_{k}(x) t^{k} .
\end{aligned}
$$

We start with selecting the initial guesses of the approximations as

$$
a_{0}(x)=f_{0}(x) \quad \text { and } \quad b_{0}(x)=f_{0}(x) .
$$

To find $a_{1}(x)$ and $b_{1}(x)$, we construct the residual functions

$$
\begin{aligned}
\operatorname{Res}_{V_{1}}^{1}(x, t)= & \frac{\partial}{\partial t}\left(f_{0}(x)+a_{1}(x) t\right)-\frac{1}{2} \sigma_{1}^{2} x^{2} \frac{\partial^{2}}{\partial x^{2}}\left(f_{0}(x)+a_{1}(x) t\right) \\
& -r_{1} x \frac{\partial}{\partial x}\left(f_{0}(x)+a_{1}(x) t\right)+r_{1}\left(f_{0}(x)+a_{1}(x) t\right) \\
& +q_{1}\left(f_{0}(x)+a_{1}(x) t-f_{0}(x)-b_{1}(x) t\right),
\end{aligned}
$$

and

$$
\begin{aligned}
\operatorname{Res}_{V_{2}}^{1}(x, t)= & \frac{\partial}{\partial t}\left(f_{0}(x)+b_{1}(x) t\right)-\frac{1}{2} \sigma_{2}^{2} x^{2} \frac{\partial^{2}}{\partial x^{2}}\left(f_{0}(x)+b_{1}(x) t\right) \\
& -r_{1} x \frac{\partial}{\partial x}\left(f_{0}(x)+b_{1}(x) t\right)+r_{2}\left(f_{0}(x)+b_{1}(x) t\right) \\
& +q_{2}\left(f_{0}(x)+b_{1}(x) t-f_{0}(x)-a_{1}(x) t\right) .
\end{aligned}
$$


Using the fact that $\operatorname{Res}_{V_{1}}^{1}(x, 0)=\operatorname{Res}_{V_{2}}^{1}(x, 0)=0$, we obtain

$$
\begin{aligned}
& a_{1}(x)=\frac{1}{2} \sigma_{1}^{2} x^{2} f_{0}^{\prime \prime}+r_{1} x f_{0}^{\prime}-r_{1} f_{0}, \\
& b_{1}(x)=\frac{1}{2} \sigma_{2}^{2} x^{2} f_{0}^{\prime \prime}+r_{2} x f_{0}^{\prime}-r_{2} f_{0} .
\end{aligned}
$$

Similarly, for $m=2$, coefficients $a_{2}(x)$ and $b_{2}(x)$ can be found from the following equations $\frac{\partial}{\partial t} \operatorname{Res}_{V_{1}}^{2}(x, 0)=0$ and $\frac{\partial}{\partial t} \operatorname{Res}_{V_{2}}^{2}(x, 0)=0$. That is,

$$
\begin{gathered}
a_{2}(x)=\frac{1}{2}\left[\frac{1}{2} \sigma_{1}^{2} x^{2} a_{1}^{\prime \prime}(x)+r_{1} x a_{1}^{\prime}(x)-a_{1}(x) r_{1}-q_{1}\left(a_{1}(x)-b_{1}(x)\right)\right], \\
b_{2}(x)=\frac{1}{2}\left[\frac{1}{2} \sigma_{2}^{2} x^{2} b_{1}^{\prime \prime}(x)+r_{2} x b_{1}^{\prime}(x)-b_{1}(x) r_{2}-q_{2}\left(b_{1}(x)-a_{1}(x)\right)\right] .
\end{gathered}
$$

Thus, by applying RPSM, we can get a general form for $a_{k}(x)$ and $b_{k}(x)$ as

$$
\begin{gathered}
a_{k}(x)=\frac{1}{k}\left[\frac{1}{2} \sigma_{1}^{2} x^{2} a_{k-1}^{\prime \prime}(x)+r_{1} x a_{k-1}^{\prime}(x)-r_{1} a_{k-1}(x)-q_{1}\left(a_{k-1}(x)-b_{k-1}(x)\right)\right] \\
b_{k}(x)=\frac{1}{k}\left[\frac{1}{2} \sigma_{2}^{2} x^{2} b_{k-1}^{\prime \prime}(x)+r_{2} x b_{k-1}^{\prime}(x)-r_{2} b_{k-1}(x)-q_{2}\left(b_{k-1}(x)-a_{k-1}(x)\right)\right]
\end{gathered}
$$

\section{Numerical investigation}

In this section we test the proposed scheme on some examples to investigate the efficiency and accuracy of the RPS method. We consider the system of coupled partial differential equations (22) with different parameters and regimes.

All computations were carry out by using of Mathematica 10 software package. Initially we price an American put option with the maturity $T=1$ and the strike $K=9$.

Example 4.1. Consider the two regime model that have no jumps between different regime, that is, $q_{i}=0,(i=1,2)$. Accordingly, we have two different and independent models.

$$
\begin{aligned}
& \frac{\partial V_{1}}{\partial t}-\frac{1}{2} \sigma_{1}^{2} x^{2} \frac{\partial^{2} V_{1}}{\partial x^{2}}-r_{1} x \frac{\partial V_{1}}{\partial x}+r_{1} V_{1}=0 \\
& \frac{\partial V_{2}}{\partial t}-\frac{1}{2} \sigma_{2}^{2} x^{2} \frac{\partial^{2} V_{2}}{\partial x^{2}}-r_{2} x \frac{\partial V_{2}}{\partial x}+r_{2} V_{2}=0
\end{aligned}
$$


subject to the initial conditions

$$
V_{1}(x, 0)=V_{2}(x, 0)=9-x, \quad i=1,2,
$$

where the parameters in the two regimes are $r_{1}=0.1, \sigma_{1}=0.8$ and $r_{2}=0.05$, $\sigma_{2}=0.3$. For numerical simulation, Table 1 and Table 2 show the approximate solution $V_{1}(x, t)$ and $V_{2}(x, t)$, respectively, of Example 4.1 compared with Runge Kutta method (RKM) containing the absolute error and relative error at $x=0.5$ and some selected grid points $t$ with step size 0.1. To show the geometric behaviors of the RPS approximation of Example 4.1, the 3-dimensional surface plots of $V_{1}(x, t)$ and $V_{2}(x, t)$ are illustrated in Figure 1 and Figure 2, respectively, for $(x, t) \in[0,1] \times[0,1]$ in comparing with RKM.

Table 1: Numerical results of $V_{1}$ in Example 4.1.

\begin{tabular}{lllll}
\hline$t$ & RKM & RPS & Absolute Error & Relative Error \\
\hline 0.0 & 8.50000 & 8.50000 & 0.00 & 0.00 \\
0.1 & 8.41045 & 8.41045 & $2.38937 \times 10^{-7}$ & $2.84096 \times 10^{-8}$ \\
0.2 & 8.32179 & 8.32179 & $9.71724 \times 10^{-7}$ & $1.16769 \times 10^{-7}$ \\
0.3 & 8.23401 & 8.23401 & $1.68492 \times 10^{-6}$ & $2.04630 \times 10^{-7}$ \\
0.4 & 8.14710 & 8.14710 & $2.29910 \times 10^{-6}$ & $2.82198 \times 10^{-7}$ \\
0.5 & 8.06106 & 8.06106 & $2.90932 \times 10^{-6}$ & $3.60910 \times 10^{-7}$ \\
0.6 & 7.97588 & 7.97588 & $3.51328 \times 10^{-6}$ & $4.40488 \times 10^{-7}$ \\
0.7 & 7.89154 & 7.89154 & $4.10882 \times 10^{-6}$ & $5.20661 \times 10^{-7}$ \\
0.8 & 7.80804 & 7.80804 & $4.69395 \times 10^{-6}$ & $6.01169 \times 10^{-7}$ \\
0.9 & 7.72538 & 7.72538 & $5.16808 \times 10^{-6}$ & $6.68974 \times 10^{-7}$ \\
1.0 & 7.64353 & 7.64354 & $5.51963 \times 10^{-6}$ & $7.22131 \times 10^{-7}$ \\
\hline
\end{tabular}

Example 4.2. Consider a special case with two identical regimes so that $r_{1}=r_{2}=0.1$ and $\sigma_{1}=\sigma_{2}=0.8$, but $q_{1}=6$ and $q_{2}=9$.

The initial conditions are the same as in Example 4.1.

In this case, there is jump between two regimes, but they are actually the same regime. Thereby, the prices in both regimes will be the same. For numerical simulation, Table 3 shows the approximate solutions $V_{1}(x, t)$ and $V_{2}(x, t)$ of Example 4.2 for $x=6$ and some selected grid points $t$ with step size 0.1 . These results demonstrate the correctness of the proposed techniques.

Example 4.3. Now, we consider the problem with two different regimes. The parameters in two regimes are $r_{1}=0.1, \sigma_{1}=0.8, q_{1}=6$ and $r_{2}=0.05$, 
Table 2: Numerical results of $V_{2}$ in Example 4.1.

\begin{tabular}{lllll}
\hline$t$ & RKM & RPS & Absolute Error & Relative Error \\
\hline 0.0 & 8.50000 & 8.50000 & 0.00 & 0.00 \\
0.1 & 8.45511 & 8.45511 & $3.00327 \times 10^{-8}$ & $3.55202 \times 10^{-9}$ \\
0.2 & 8.41045 & 8.41045 & $1.22700 \times 10^{-7}$ & $1.45890 \times 10^{-8}$ \\
0.3 & 8.36601 & 8.36601 & $2.13805 \times 10^{-7}$ & $2.55563 \times 10^{-8}$ \\
0.4 & 8.32179 & 8.32179 & $2.93187 \times 10^{-7}$ & $3.52312 \times 10^{-8}$ \\
0.5 & 8.27779 & 8.27779 & $3.72816 \times 10^{-7}$ & $4.50381 \times 10^{-8}$ \\
0.6 & 8.23401 & 8.23401 & $4.52359 \times 10^{-7}$ & $5.49379 \times 10^{-8}$ \\
0.7 & 8.19045 & 8.19045 & $5.31490 \times 10^{-7}$ & $6.48914 \times 10^{-8}$ \\
0.8 & 8.14710 & 8.14710 & $6.09888 \times 10^{-7}$ & $7.48595 \times 10^{-8}$ \\
0.9 & 8.10398 & 8.10398 & $6.74305 \times 10^{-7}$ & $8.32067 \times 10^{-8}$ \\
1.0 & 8.06106 & 8.06106 & $7.22957 \times 10^{-7}$ & $8.96850 \times 10^{-8}$ \\
\hline
\end{tabular}

Table 3: Numerical results of $V_{1}$ and $V_{2}$ in Example 4.2.

\begin{tabular}{ccc}
\hline$t$ & Appr. sol. $V_{1}$ & Appro. sol. $V_{2}$ \\
\hline 0 & 2.80364 & 2.80364 \\
0.1 & 2.88548 & 2.88548 \\
0.2 & 2.96815 & 2.96815 \\
0.3 & 3.05164 & 3.05164 \\
0.4 & 3.13598 & 3.13598 \\
0.5 & 3.22116 & 3.22116 \\
0.6 & 3.30720 & 3.30720 \\
0.7 & 3.39410 & 3.39410 \\
0.8 & 3.48188 & 3.48188 \\
0.9 & 3.57054 & 3.57054 \\
1 & 3.6601 & 3.6601 \\
\hline
\end{tabular}

$\sigma_{2}=0.3, q_{2}=9$. For numerical simulation, Table 4 and Table 5 show the approximate solution $V_{1}(x, t)$ and $V_{2}(x, t)$, respectively, of Example 4.3 for $x=6$ and some selected grid points $t$ with step size 0.1. Calculations are performed for $T-t$. Also, the absolute error is calculated. In our problem the exact solution is unknown. In this case, the absolute error is defined as follows:

$$
A E\left(V_{i}(x, t)\right)=\left|V_{i}^{k+1}(x, t)-V_{i}^{k}(x, t)\right|,
$$

or, by direct substitution into Equation (22).

The surface plots of the option prices are presented in Fig. 3 and Fig .4 as 


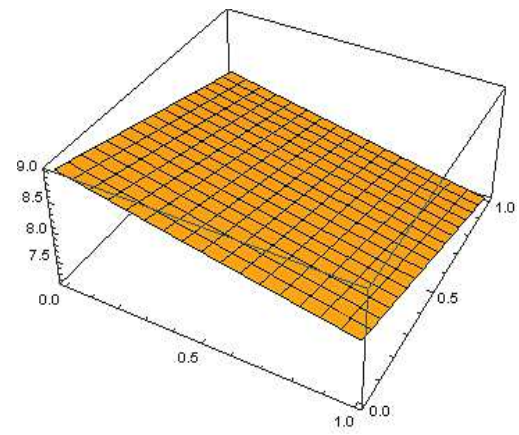

(a)

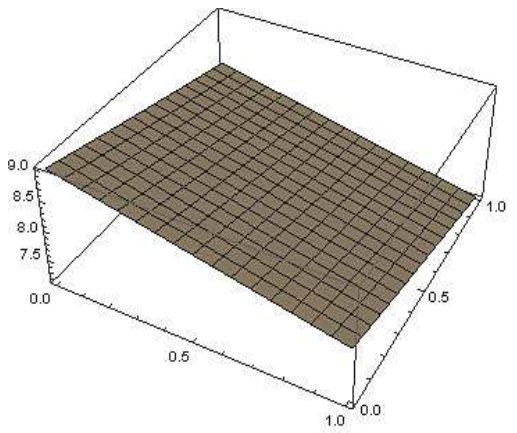

(b)

Figure 1: Surface plot of the RPS approximation of $V_{1}$ for Example 4.1 with $x \in[0,1]$ and $t \in[0,1]$ : (a) RKM (b) RPS.

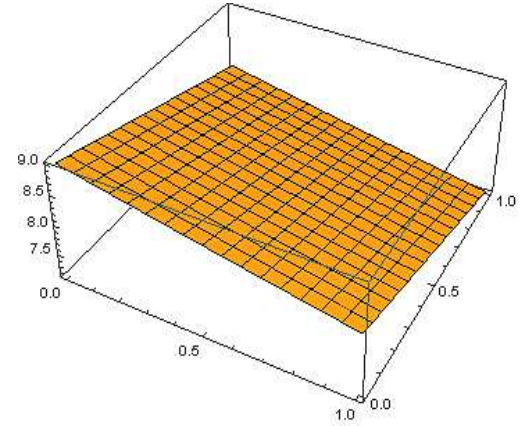

(a)

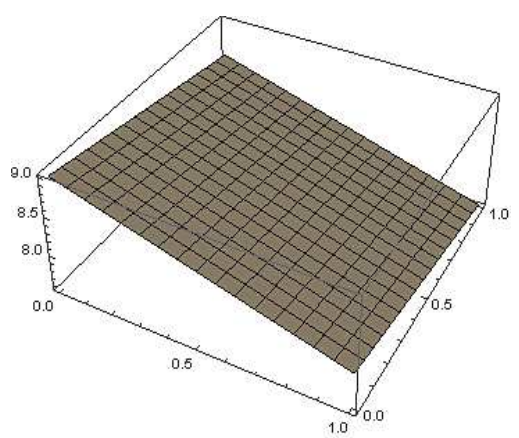

(b)

Figure 2: Surface plot of the RPS approximation of $V_{2}$ for Example 4.1 with $x \in[0,1]$ and $t \in[0,1]:$ (a) RKM (b) RPS.

function of $x$ and $t$. Both figures produce smooth surfaces. This confirms the reliability of the method.

\section{Conclusion and remarks}

In this paper, the residual power series method has been proposed and applied successfully for solving a class of coupled systems of PDEs arising in the option pricing problem with regime switching. The analytic solutions in a form of convergent series are obtained. Models with different parameters and regimes have been used to test the validity and reliability of the present technique. 
Table 4: Numerical results of $V_{1}$ in Example 4.3

\begin{tabular}{ccc}
\hline$t$ & Appr. sol. $V_{1}$ & Abs. error \\
\hline 0 & 7.30809 & 0.00154695 \\
0.1 & 5.94422 & 0.000491357 \\
0.2 & 4.97776 & 0.000136545 \\
0.3 & 4.30554 & 0.0000320468 \\
0.4 & 3.85126 & $6.0335 \times 10^{-6}$ \\
0.5 & 3.55858 & $8.4181 \times 10^{-7}$ \\
0.6 & 3.38591 & $7.6307 \times 10^{-8}$ \\
0.7 & 3.30261 & $3.52307 \times 10^{-9}$ \\
0.8 & 3.28608 & $4.85691 \times 10^{-11}$ \\
0.9 & 3.31967 & $4.08562 \times 10^{-14}$ \\
1 & 3.39114 & $8.88178 \times 10^{-16}$ \\
\hline
\end{tabular}

Table 5: Numerical results of $V_{2}$ in Example 4.3

\begin{tabular}{ccc}
\hline$t$ & Appr. sol. $V_{2}$ & Abs. error \\
\hline 0 & 10.1517 & 0.00262781 \\
0.1 & 7.99648 & 0.000844615 \\
0.2 & 6.44544 & 0.000238202 \\
0.3 & 5.34136 & 0.0000569705 \\
0.4 & 4.56791 & 0.0000109977 \\
0.5 & 4.03917 & $1.58865 \times 10^{-6}$ \\
0.6 & 3.69173 & $1.51612 \times 10^{-7}$ \\
0.7 & 3.47883 & $7.61452 \times 10^{-9}$ \\
0.8 & 3.36602 & $1.23492 \times 10^{-10}$ \\
0.9 & 3.32788 & $1.58096 \times 10^{-12}$ \\
1 & 3.34571 & $1.77636 \times 10^{-15}$ \\
\hline
\end{tabular}

Therefore, it can be concluded that the RPS method is effective, accurate and simple in application.

\section{References}

[1] M. Alaroud, M. Al-Smadi, R.R. Ahmad and U.K. Salma Din, Computational optimization of residual power series algorithm for certain classes of fuzzy fractional differential equations, Internat. J. of Differential Equations, 2018 (2018), Art. ID 8686502, 1-11. 


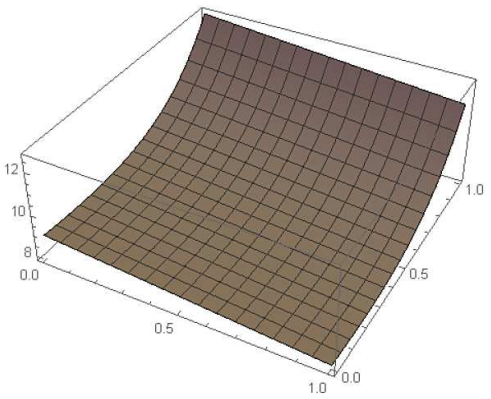

Figure 3: Surface plot of the RPS approximation of $V_{1}$ for Example 4.3

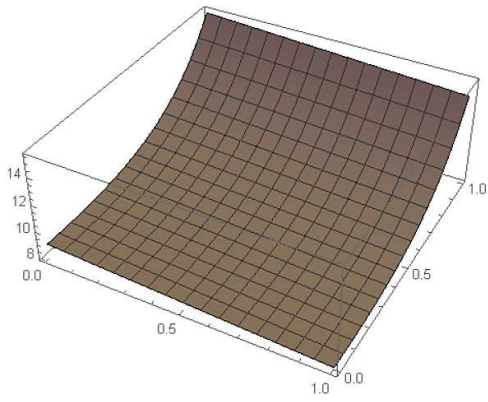

Figure 4: Surface plot of the RPS approximation of $V_{2}$ for Example 4.3

[2] R. Abu-Gdairi, M. Al-Smadi and G. Gumah, An expansion iterative technique for handling fractional differential equations using fractional power series scheme, J. of Math. and Stat., 11, No 2 (2015), 29-38.

[3] M.Al-Smadi, Solving fractional system of partial differential equations with parameters derivative by combining the GDTM and RDTM, Nonlinear Studies, 26, No 3 (2019), 587-601.

[4] M. Al-Smadi and O. Abu Arqub, Computational algorithm for solving fredholm time-fractional partial integrodifferential equations of dirichlet functions type with error estimates, Appl. Math. and Comput., 342 (2019), 280-294.

[5] S. Alshammari, M. Al-Smadi, I. Hashim and M.A. Alias, Residual Power Series Technique for Simulating Fractional BagleyTorvik Problems 
Emerging in Applied Physics, Applied Sciences, 9, No 23 (2019), 5029; doi:10.3390/app9235029.

[6] F. Black, M. Scholes, The pricing of options and corporate liabilities, J. of Political Economy, 81 (1973), 637-654.

[7] J. Buffington, R. Elliot, American options with regime switching, Intern. J. of Theoret. and Appl. Finance, 5 (2002), 497-514.

[8] C. Chiarella, C. Nikitopoulos, E. Schlogl and H. Yang, Pricing American options under regime switching using method of lines, Quantitative Finance Research Center (2016), Research Paper 368.

[9] C. Christara and D.M. Dang, Adaptive and high-order methods for valuing American options, J. of Comput. Finance, 14 (2011), 73113.

[10] D. M. Dang, Adaptive Finite Difference Methods for Valuing American Options, M.Sc. Thesis, Dept. of Computer Science, University of Toronto, Canada, 2007.

[11] P.A. Forsyth and K.R. Vetzal, Quadratic convergence for valuing American options using a penalty method, SIAM J. on Scientific Computing, 23 (2002), 20952122.

[12] A. Freihat, S. Hasan, M. Al-Smadi, M. Gaith and S. Momani, Construction of fractional power series solutions to fractional stiff system using residual functions algorithm, Advances in Difference Equations, 2019 (2019), \# 95; doi:10.1186/s13662-019-2042-3

[13] S. Hasan, A. Al-Zoubi, A. Freihat, M. Al-Smadi and S. Momani, Solution of the fractional SIR epidemic model using the residual power series method, Appli. Math. \& Information Sci., 13, No 2 (2019), 153-161.

[14] M.S. Horng, T.L. Horng and C.Y. Tien, A method-of-lines approach for solving American Option problems, Taiwanese J. of Math., 23, No 5 (2019), 1253-1270.

[15] A.Q. Khaliq, R.H. Liu, New numerical scheme for pricing American option with regime-switching, Intern. J. of Theoret. and Appl. Finance, 12, No 3 (2009), 319-340.

[16] I. Komashynska, M. Al-Smadi, O. Abu Arqub and S. Momani, An efficient analytical method for solving singular initial value problems of nonlinear systems, Appl. Math. \& Information Sci., 10, No 2 (2016), 647-656. 
[17] I. Komashynska, M. Al-Smadi, A. Ateiwi and S. Al-Obaidy, Approximate analytical solution by residual power series method for system of fredholm integral equations, Appl. Math. \& Information Sci., 10, No 3 (2016), 975985.

[18] I. Komashynska, M. Al-Smadi, A. Al-Habahbeh and A. Ateiwi, Analytical approximate solutions of systems of multi-pantograph delay differential equations using residual power-series method, Australian J. of Basic and Appl. Sciences, 8, No 10 (2014), 664-675.

[19] R.H. Liu, Q. Zang, and G.Yin, Option pricing in a regime switching model using the Fast Fourier Transform, J. of Appl. Math. and Stoch. Anal. (2006), Art. ID 18109, 1-22.

[20] K. Moaddy, M. Al-Smadi and I. Hashim, A novel representation of the exact solution for differential algebraic equations system using residual power-series method, Discrete Dynamics in Nature and Society, 2015 (2015), Art. ID 205207, 1-12.

[21] R. Saadeh, M. Alaroud, Al-Smadi, R.R. Ahmad and U.K. Salma Din, Application of fractional residual power series algorithm to solve NewellWhiteheadSegel equation of fractional order, Symmetry, 11, No 12 (2019), 1431; doi:10.3390/sym11121431.

[22] M. Shqair, M. Al-Smadi, S. Momani and E. El-Zahar, Adaptation of conformable residual power series scheme in solving nonlinear fractional quantum mechanics problems, Appl. Sciences, 10, No 3 (2020), 890; doi:10.3390/app10030890.

[23] K. Zhang, K.L. Teo and M.Swartz, A robust numerical scheme for pricing american options under regime switching based on penalty method, Comput. Econ., 43 (2014), 463-483.

[24] O. Zhylyevskyy, A Fast Fourier Transform technique for pricing American options under stochastic volatility, Review of Derivatives Research, 13 (2010), 1-24. 